\title{
Reviewers for The Journal of the American Board of Family Practice in 1998
}

The following individuals participated in the peer review of manuscripts submitted to this journal during 1998. Their contributions are gratefully acknowledged.

Acheson, Louise

Acosta, David

Alexander, Elizabeth

Allen, Deborah

Baird, Mac

Baldwin, Laura-Mae

Barringer, Tom

Bauman, Kay

Berg, Alfred O.

Bergman, James J.

Bluestein, Daniel

Brown, Richard

Brucker, Paul

Campbell, Thomas

Carmichael, Kevin

Cass, Alvah

Colwill, Jack

Coombs, John

Crouch, Michael

Culpepper, Larry

David, Allen

Davidson, Robert C.

Demers, Raymond

Dennehy, Cathi

Derksen, Daniel J.

Deyo, Richard

Dobie, Sharon

Dong, Betty

Ebell, Mark

Eiff, Patrice

Ellsworth, Allan

Ewigman, Bernard
Ferrer, Robert

Fields, Karl

Fowkes, William

Frame, Paul

Franks, Peter

Frey, Keith

Ganiats, Ted

Garry, Joseph

Goldbaum, Gary

Green, Larry

Gravdal, Judith

Hachey, David

Heidrich, Fred

Herter, Christian

Hume, Anne

Hunt, Vincent

Katon, Wayne

Lawless, Cara

LeFevre, Michael

Losh, David

MacFarlane, Lori

Marek, Tamara

Mengel, Mark

Midtling, John

Nesbitt, Thomas S.

Newkirk, Gary

Norris, Thomas

Nuovo, James

Phillips, Ted

Phillips, William

Prislin, Michael

Rabinowitz, Howard
Ramsey, Scott

Realini, Janet

Reichel, William

Richardson, Michael

Robertson, William O.

Rodney, Wm. MacMillan

Rodriguez, Glenn S.

Rosenfeld, Joanne

Rosenblatt, Roger

Ruane, Thomas

Saultz, John

Scherger, Joseph

Schneeweiss, Ronald

Schwenk, Thomas

Shane-McWhorter, Laura

Sloane, Philip

Smith, Mindy Ann

Spach, David

Stephens, G. Gayle

Sugarman, Jonathan

Sullivan, Sean

Swee, David

Taplin, Stephen

Thom, David

Tsourounis, Candy

Walker, Edward

Wall, Eric

Williamson, Harold

Wolf, Fred

Woodhouse, William

Young, Paul 